\title{
Synaptic interactions and inhibitory regulation in auditory cortex
}

\author{
Caitlin E. Askew ${ }^{1}$ and Raju Metherate ${ }^{1}$ \\ ${ }^{1}$ Department of Neurobiology and Behavior \\ Center for Hearing Research \\ University of California, Irvine \\ Irvine, CA 92697 USA
}

\section{Correspondence}

Raju Metherate, Ph.D.

Department of Neurobiology and Behavior

University of California, Irvine

2205 McGaugh Hall

Irvine, California 92697-4550

USA

phone: (949) 824-6141

fax: (949) 824-2447

email: raju.metherate@uci.edu 


\begin{abstract}
This Special Issue focuses on the auditory-evoked mismatch negativity (MMN), an electrophysiological index of change, and its reduction in schizophrenia. The following brief review is an attempt to complement the behavioral and clinical contributions to the Special Issue by providing basic information on synaptic interactions and processing in auditory cortex. A key observation in previous studies is that the MMN involves activation of cortical $N$-methyl-Daspartate (NMDA) receptors. Yet, NMDA receptor activation is regulated by a number of synaptic events, which also may contribute to the MMN reduction in schizophrenia. Accordingly, this review will focus on synaptic interactions, notably inhibitory regulation of NMDA receptor-mediated activity, in auditory cortex.
\end{abstract}

\title{
Keywords
}

auditory cortex; glutamate; NMDA; GABA; thalamocortical; nicotinic acetylcholine receptor; mismatch negativity; synaptic integration; neuromodulation

\section{Acknowledgments}

The authors' research is supported by grants from the National Institutes of Health, USA (NIDCD R01 DC013200, P30 DC08369, T32 DC010775) and the National Science Foundation, USA (DGE-1321846). 


\section{Introduction}

The purpose of this brief review is to complement the core papers in this Special Issue on the neurobiology of the mismatch negativity $(\mathrm{MMN})$ and its reduction in schizophrenia, by providing basic information on synaptic interactions and relevant circuitry in auditory cortex. While the mechanisms and functions of the MMN are being actively investigated, as discussed in companion reviews, a key finding is that the $\mathrm{MMN}$ is reduced by drugs that block glutamate receptors of the $N$-methyl-D-aspartate (NMDA) subtype (Catts, Lai, Weickert, Weickert, \& Catts, 2015; Harms, 2015; Javitt, 2000; Javitt, Steinschneider, Schroeder, \& Arezzo, 1996; Todd et al., 2014; Wacongne, 2015). The neural origins of the MMN within the auditory system also are addressed by reviews of stimulus-specific adaptation (SSA), whereby the auditory system responds more strongly to rare or novel stimuli than to common stimuli (Ayala, Perez-Gonzalez, \& Malmierca, 2015; Grimm, Escera, \& Nelken, 2015). In turn, this review will focus on even more fundamental processes in auditory cortex, specifically, how excitatory postsynaptic potentials (EPSPs), including those mediated by NMDA receptors, are regulated by synaptic inhibition (inhibitory postsynaptic potentials, IPSPs) in auditory cortex.

Underlying every complex event like the MMN in auditory cortex and elsewhere is the integration of multiple synaptic events. That is, various excitatory, inhibitory and neuromodulatory processes integrate to determine the output of a neuron and the circuits to which it belongs. Thus, to understand synaptic integration is to better understand the mechanism underlying any form of auditory processing. A key principle is that different forms of inhibition precisely subserve different regulatory functions, and it is on this topic that we begin.

\section{Synaptic inhibition precisely regulates excitation in auditory cortex}

It is well established that an auditory stimulus (pure tone burst) elicits a short-latency EPSP in the input layers of primary auditory cortex, followed a few milliseconds later by a longer-lasting hyperpolarization consisting of one or more IPSPs (Ribaupierre, Goldstein, \& Yeni-Komshian, 1972). The rapid-onset inhibition allows for strict regulation of the neuron's firing and provides a mechanism for precise temporal processing, including the characteristic cortical "onset response" (Tan, Zhang, Merzenich, \& Schreiner, 2004; Wehr \& Zador, 2003, 
2005). A number of studies break down this response into its basic synaptic units. Electrical stimulation of thalamic inputs can produce a three-component response in sensory cortex, consisting of an early EPSP, an early IPSP, and a late IPSP (Fig. 1) (Douglas \& Martin, 1991; Metherate \& Ashe, 1993; Purpura \& Shofer, 1964; Purpura, Shofer, \& Musgrave, 1964). Under some conditions, such as repetitive or paired-pulse stimulation, a fourth component, or late EPSP, is revealed (Fig. 2) (Cox, Metherate, Weinberger, \& Ashe, 1992; Metherate \& Ashe, 1994). Despite broad temporal overlap in their latencies and durations, each of these four synaptic potentials depends on a specific neurotransmitter receptor sub-type with unique ion permeability. In order of latency, the four synaptic potentials are: 1) an early EPSP mediated by AMPA receptors permeable to cations, 2) an early IPSP mediated by GABA-A receptors permeable to $\left.\mathrm{Cl}^{-}, 3\right)$ a late EPSP mediated by NMDA receptors permeable to cations, notably $\mathrm{Ca}^{2+}$, and 4) a late IPSP mediated by metabotropic GABA-B receptors that activate a $\mathrm{K}^{+}$channel (Connors, Malenka, \& Silva, 1988; Cox, et al., 1992; Metherate \& Ashe, 1994, 1995; Sutor \& Hablitz, 1989).
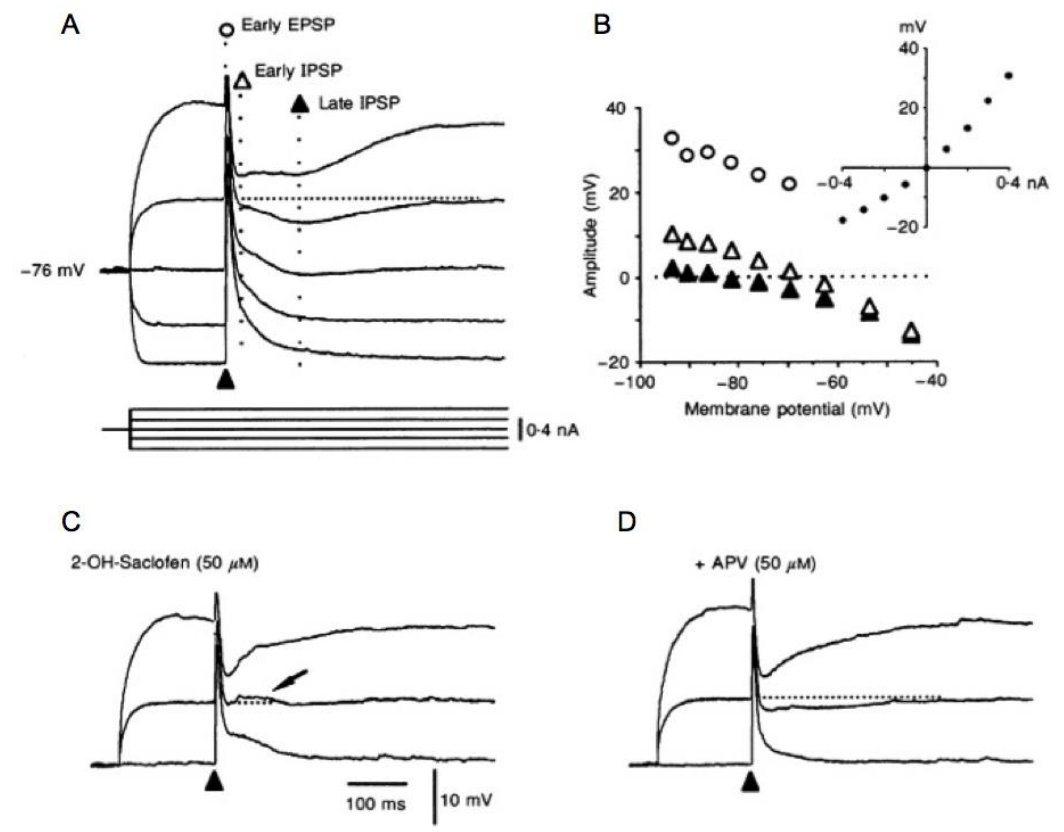

D

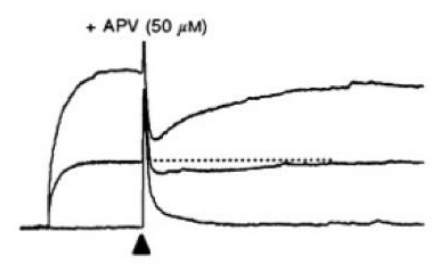

Figure 1. The stereotypic synaptic response in auditory cortex evoked by afferent stimulation in vitro comprises a glutamatergic EPSP and two GABAergic IPSPS. A: the neuron is hyperpolarized or depolarized from rest via current from the patch pipette (current record under first set of traces; triangle above current record indicates time of electrical stimulus). B: shows 
the amplitude of each response component vs. membrane potential, and the inset shows neuron's input resistance. C,D: Reduction of late-IPSP with the GABA-B antagonist 2-OH saclofen reveals late-EPSP (arrow in C) that is reduced by NMDA receptor antagonist APV (D). Scales in C apply to all traces. From (Metherate \& Ashe, 1994).

The NMDA receptor-mediated late EPSP can be suppressed by both early and late IPSPs, presumably because IPSP-mediated hyperpolarization prevents activation of the NMDA receptor, but can be "released" under conditions that reduce the efficacy of the IPSPs, such as during paired-pulse stimulation (Fig. 2) (Metherate \& Ashe, 1994). Thus, at this most basic level of synaptic interaction, inhibition regulates excitation, notably NMDA receptor-mediated synaptic activity. This theme is repeated at both the single-neuron, and broader neural-systems, levels (see below).

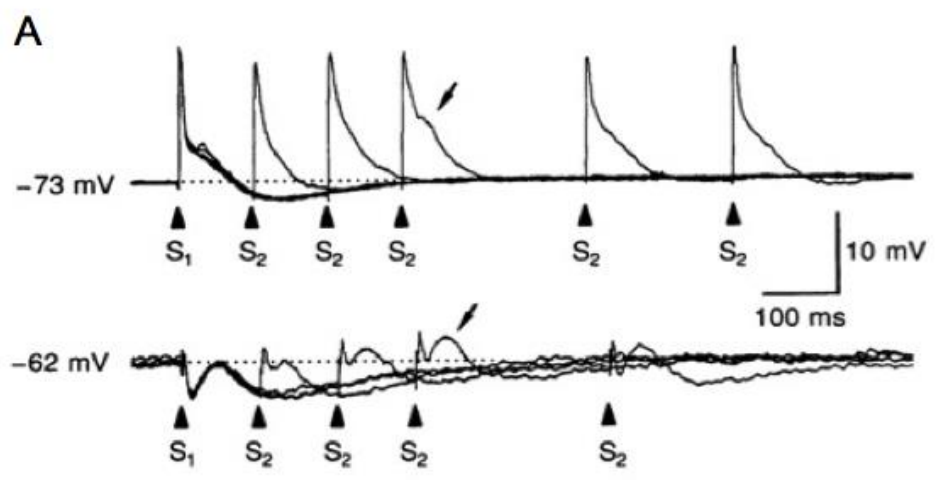

B

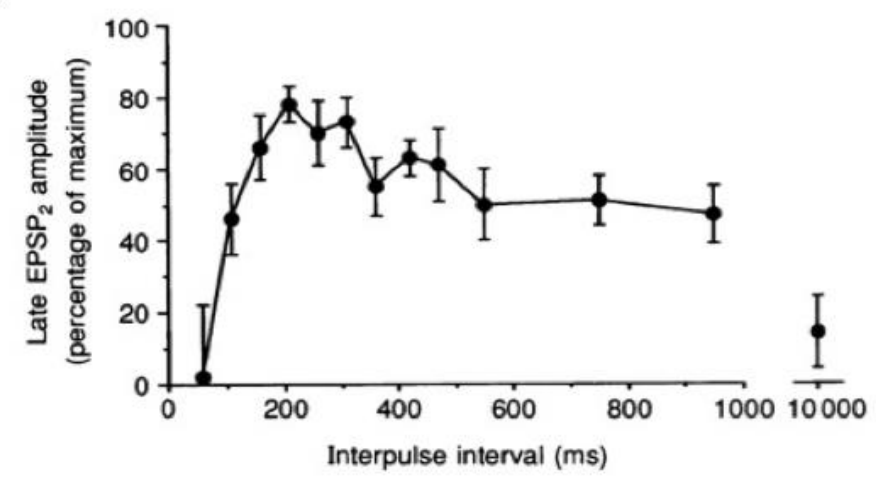

Figure 2. Facilitation of NMDA receptor-mediated late-EPSP by paired pulse electrical stimulation, in vitro. $S_{1}$ is first stimulus, $S_{2}$ is second stimulus delivered at various interpulse intervals after the first. Traces (A) are from two different neurons exhibiting different resting 
membrane potentials, with 4-5 trials overlaid. Arrows point to optimally enhanced late-EPSP for each neuron. (B) Group data showing amplitude of enhanced late-EPSP at various interpulse intervals. From (Metherate \& Ashe, 1994)

Many of the experiments giving rise to these results were performed using electrical stimulation of in vitro brain slice preparations. Yet, the functional consequences of inhibitory regulation for auditory processing are easily observed in vivo, in response to acoustic stimulation. An important example is forward masking, whereby auditory stimuli can suppress responsiveness to subsequent stimuli for hundreds of milliseconds (Brosch \& Schreiner, 1997; Calford \& Semple, 1995; Sutter \& Loftus, 2003; Wehr \& Zador, 2005). In vivo intracellular studies confirm that inhibition follows excitation quickly, but the studies differ on the exact mechanism of long-lasting hyperpolarization and forward masking. One study using in vivo whole cell recordings found that hyperpolarization and suppression are likely accounted for by synaptic inhibition (as in vitro, Fig. 1), rather than non-synaptic forms of inhibition such as an intrinsic potassium conductance (Tan, et al., 2004). Another study using similar methods found that tone-evoked inhibitory synaptic conductances rarely last longer than 50-100 ms (Wehr \& Zador, 2005), significantly shorter than the duration of spike suppression that can last for over $500 \mathrm{~ms}$. The latter authors suggest that synaptic inhibition accounts for the first $100 \mathrm{~ms}$ or so of spike suppression, but longer-lasting effects likely involve synaptic depression resulting in reduced pre-synaptic neurotransmitter release. Although they demonstrate the existence of a GABA-B receptor mediated conductance that lasts 200-300 ms (i.e., the late IPSP), they conclude that the small magnitude of this current may not fully account for long-lasting suppression.

Regardless of whether synaptic inhibition is the primary cause of forward masking or only a contributing factor, it appears that synaptic excitation and inhibition have approximately similar frequency tuning (Tan, et al., 2004; Wehr \& Zador, 2003). This “co-tuning” or "balanced" excitation and inhibition refers to the similarity in time course and amplitude of excitatory vs. inhibitory responses over a wide spectral range of stimuli (Wehr \& Zador, 2003; Zhao et al., 2015). Nevertheless, co-tuning is only approximate, and in fact may be "unbalanced" (more inhibitory than excitatory) at the edges of a neuron's receptive field (Wu, Arbuckle, Liu, Tao, \& Zhang, 2008). Heightened inhibition at the receptive field edge may underlie the lateral 
inhibition identified in numerous extracellular studies using forward masking procedures (Calford \& Semple, 1995; Pantev et al., 2004; Suga, 1995), and demonstrates a potential role for inhibition in sharpening frequency tuning. Excitatory and inhibitory balance also breaks down near acoustic threshold, where excitatory and inhibitory inputs to a neuron can exhibit different thresholds and frequency tuning (Zhao, et al., 2015). Thus, although excitation and inhibition are approximately co-tuned, there are fine differences that likely play an important role in auditory processing.

Synaptic inhibition also may be necessary for acoustic-evoked gamma fluctuations-i.e., oscillation of local field potentials in the gamma range, including $\sim 40 \mathrm{~Hz}$ - in auditory cortex, although the evidence to date is largely indirect. Studies in other brain regions have linked IPSPs to the production of gamma oscillations (Penttonen, Kamondi, Acsady, \& Buzsaki, 1998; Wang \& Buzsaki, 1996), and local field recordings in auditory cortex detect a burst of gamma band activity in response to tones (Vianney-Rodrigues, Iancu, \& Welsh, 2011). Electrical thalamic stimulation of the in vitro thalamocortical slice preparation elicits a short latency EPSP in auditory cortex followed by polysynaptic (i.e., network or ensemble) activity that resembles gamma oscillations riding on a slow potential or late EPSP (Fig. 3) (Metherate \& Cruikshank, 1999). The gamma fluctuations appear to consist of rapid alternation of depolarizations and $\mathrm{Cl}^{-}-$ mediated hyperpolarizations, i.e., likely EPSPs and IPSPs. This network activity propagates across cortex and has been hypothesized to resemble neuronal ensembles that encode auditory information in vivo. Several observations support the idea that the ensemble activity reflects a slow (NMDA receptor-mediated) EPSP with superimposed gamma oscillations, which together may contribute, in vivo, to long-duration and long-latency potentials such as the MMN. The ensemble activity appears in an all-or-none manner during stimulation near threshold intensities, and can not be elicited at stimulation rates faster than once every 10-15 seconds (i.e., it exhibits a refractory period). Similarly, NMDA receptor antagonists reduce the ensemble activity abruptly and completely, as if the excitation required to activate it falls below a threshold value. This interpretation implies only that the triggering excitation is glutamatergic (presumably, the late EPSP), and does not contradict evidence that the gamma oscillation contains GABAergic IPSPs. The function of this in vitro ensemble response is not clear, and notably has not yet been tested in vivo, but in terms of its latency, time course, sensitivity to repetition rate and sensitivity to glutamate antagonists, it resembles event-related potentials, such as the MMN, that contribute 
to the detection of novel or behaviorally significant stimuli (Ehlers, Kaneko, Wall, \& Chaplin, 1992; Javitt, et al., 1996; Rugg \& Coles, 1995).

Future studies must bridge the gap between these cellular studies, including inhibitory regulation of NMDA receptor-mediated excitation, and the MMN in behaving animals.
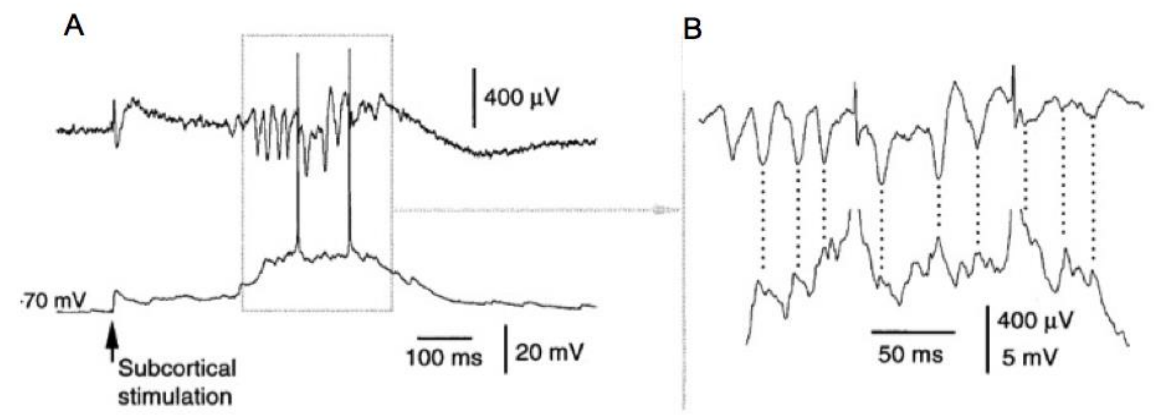

Figure 3. Afferent stimulation that activates the NMDA receptor-mediated EPSP can trigger high-frequency (gamma) cortical activity in the auditory thalamocortical slice. Top trace is local field potential, bottom trace is simultaneous intracellular recording within the same cortical "column." Traces in (B) are enlarged from the box in (A) and show correspondence between extracellular gamma-range oscillations and intracellular membrane potential fluctuations. In particular, extracellular negative potentials tend to correspond to intracellular depolarizations (vertical dotted lines), suggesting that both reflect fast EPSPs; similarly, intervening extracellular positivities and intracellular hyperpolarizations may reflect fast IPSPs. From (Metherate \& Cruikshank, 1999).

A final caveat is that in vivo studies to date generally have not distinguished between the type of inhibition elicited by tones at a neuron's characteristic frequency (CF) vs. stimuli that are spectrally distant from CF (e.g., that produce lateral inhibition). CF tones activate feed-forward excitation and inhibition that are well characterized in vivo and in vitro, whereas spectrally distant stimuli activate intracortical, "horizontal" pathways (Happel, Jeschke, \& Ohl, 2010; Kaur, Lazar, \& Metherate, 2004; Metherate et al., 2005). It is likely that these distinct cortical circuits activate different types of inhibition, and future studies will need to address this issue.

As the studies reviewed above show, at the most fundamental level of auditory processing, inhibition precisely regulates excitation to provide both rapid and long-lasting 
control of synaptic integration that undoubtedly is central to complex auditory responses. The neural circuitry contributing to such responses will be discussed next.

\section{GABAergic interneurons in cortical circuits}

In addition to understanding the importance of inhibitory regulation to synaptic integration, it is necessary to understand how different inhibitory interneurons contribute to auditory processing at the level of cortical circuitry. The neural circuits intrinsic to auditory cortex include excitatory pyramidal neurons that release glutamate, and inhibitory non-pyramidal interneurons that release GABA. These circuits respond to a variety of inputs from thalamic relay nuclei and other cortical regions to process auditory information, the result of which underlies auditory perception. An understanding of the functional connectivity among different neuron types within cortical circuits is an important step towards understanding mechanisms of cortical processing. As knowledge in this area has exploded in recent years with the introduction of new molecular and genetic tools, it quickly has become clear that GABAergic interneurons are an essential and diverse regulatory component in auditory cortex circuitry.

GABAergic inhibitory interneurons comprise about $15-25 \%$ of all neurons in cortex (DeFelipe et al., 2013; Rudy, Fishell, Lee, \& Hjerling-Leffler, 2010). They are found in all layers of the cortex, where they form a minority of cells in each layer with the exception of layer 1 ; although layer 1 is cell-sparse, containing mostly the axons and dendrites of excitatory neurons whose cell bodies lie elsewhere, nearly all cells intrinsic to layer 1 are GABAergic. Inhibitory interneurons can be directly activated by thalamocortical axons or inputs from other cortical areas, but the pattern of responding to inputs varies according to interneuron subtype (Kepecs \& Fishell, 2014). Interneuron outputs are similarly specific; notably, they can target either the soma or dendrites of other neurons, which is an important determinant of function. Direct inhibitory projections to the soma or axon initial segment exert powerful inhibition of neuron output, while projections to distal dendrites are presumably more modulatory. Projection patterns can vary according to interneuron subtype, or be mixed within groups, depending on the method of interneuron classification (Kepecs \& Fishell, 2014).

Recent in vitro mapping of connectivity between pyramidal cells and interneurons in the input layer of mouse auditory cortex reveals that connection probability is distance dependent; 
i.e., nearby cells have a greater probability of being connected (Levy \& Reyes, 2012). The dropoff in connectivity with distance is similar for both pyramidal-pyramidal and pyramidalinterneuron connections, but the strength of pyramidal-interneuron connections is greater, reinforcing the important position occupied by inhibitory interneurons in cortical circuitry (Cruikshank, Urabe, Nurmikko, \& Connors, 2010; Levy \& Reyes, 2012).

Inhibitory interneurons can be categorized in non-overlapping groups based on molecular markers, notably the calcium binding protein parvalbumin ( $\mathrm{PV}$ interneurons), and the peptides somatostatin (SOM interneurons) and vasoactive intestinal polypeptide (VIP interneurons). The majority of cortical interneurons fall into one of these three groups, although a remaining $\sim 20 \%$ do not express any of these markers (Xu, Roby, \& Callaway, 2010). Other methods for classifying interneurons exploit morphological and physiological features, and the resulting classification schemes overlap with molecular-based schemes (Markram et al., 2004). These three interneuron classes (PV, SOM, VIP) appear to have distinct functions and connectivity. For example, PV interneurons mediate feed-forward inhibition and can enhance functional connectivity in cortical (vertical) columns, but not lateral or feedback (horizontal) connections within layers (Hamilton et al., 2013). Activation of PV neurons strongly suppresses spontaneous firing and weakly suppresses stimulus-evoked firing, producing an overall enhancement of afferent input "signal to noise" ratio. Conversely, SOM neurons in the superficial layers of visual cortex are excited by horizontal projections in the same layer, contributing to surround suppression in layer 2/3 pyramidal neurons (Adesnik \& Scanziani, 2010). If SOM neurons act similarly in auditory cortex, they might preferentially mediate lateral inhibition and regulate receptive field breadth.

PV cells also have broader frequency receptive fields and faster response onset than SOM cells, while the narrower tuning of SOM cells is more similar to that of excitatory pyramidal neurons (Li et al., 2014). This differential tuning and latency are consistent with the notion that PV neurons mediate robust feed-forward inhibition. Anatomical evidence agrees with this idea, as PV cells project more locally than do SOM cells and often target neuronal somata, allowing for powerful and temporally precise effects (Yuan, Fink, Winer, \& Schreiner, 2011). In contrast, SOM cells more often target distal dendrites of pyramidal neurons, and their slower time course allows for integration of inputs over a broader time frame (Di Cristo et al., 2004; Pouille \& Scanziani, 2001). 
Interestingly, VIP neurons recently were shown to have a disinhibitory role in auditory cortex (Pi et al., 2013). That is, they project to and inhibit interneurons that, in turn, project to pyramidal neurons, with the end result being that activation of VIP neurons reduces inhibition on pyramidal cells. VIP neurons may preferentially inhibit SOM interneurons, but also regulate some PV interneurons. An auditory discrimination task strongly and preferentially triggered VIP neuron-dependent disinhibition, demonstrating behavioral relevance.

Across all subtypes, GABAergic inhibitory interneurons exhibit complex connectivity and functional specificity in their control of cortical excitatory output. Although it seems unlikely that specific interneuron subtypes are associated with specific IPSP subtypes (e.g., Fig. 1), this question will only be fully resolved by future studies.

\section{Neuromodulatory regulation of inhibitory neurons}

In addition to the importance of inhibitory regulation in auditory cortex, it is just as important to acknowledge factors that regulate inhibition itself. These factors include modulatory actions of neurotransmitters; i.e., neuromodulation. As distinct from the fast actions of neurotransmitters that mediate sensory input, such as glutamatergic excitation mediated by AMPA receptors and GABAergic inhibition mediated by GABA-A receptors, neuromodulation can be broadly defined as slower, lasting changes in neuronal excitability that depend on intracellular biochemical signaling (Kaczmarek \& Levitan, 1987). Typical neuromodulatory actions involve metabotropic G-protein coupled receptors and downstream signal transduction such as that activated by acetylcholine (via muscarinic receptors), norepinephrine, dopamine and serotonin. However, intracellular signaling also can be triggered by activation of ionotropic receptors with $\mathrm{Ca}^{2+}$ permeability, including NMDA receptors and some nicotinic acetylcholine receptor (nAChR) subtypes (Broide \& Leslie, 1999; McGehee \& Role, 1995). Regardless of their source, neuromodulatory actions are relatively slow and long-lasting, and their effects can in turn alter neural responsiveness for prolonged periods. Neuromodulation of forebrain neurons is likely responsible for altered neural activity during states of arousal and attention, and modulate the processing of sensory information accordingly.

Since this Special Issue is focused on schizophrenia research, and given the high rate of smoking among schizophrenics, it is relevant to note that neuromodulatory actions of nicotine 
affect cortical interneurons differentially. There is substantial evidence that nicotine overtly excites some inhibitory interneurons and produces more subtle effects in other neurons. In hippocampus, nicotine can selectively excite interneurons but not pyramidal neurons (Frazier et al., 1998). In prefrontal cortex, nAChRs are found on both inhibitory interneurons and pyramidal cells, and in both cases can be located at presynaptic terminals or on postsynaptic membranes, but nicotine-induced overt neuronal firing occurs only in interneurons (Poorthuis et al., 2012). In other neocortical areas, nAChR agonists selectively affect specific interneuron subtypes, for example producing excitation of VIP interneurons but no effect on PV or SOM interneurons (Porter et al., 1999), or non-fast-spiking interneurons (Gulledge, Park, Kawaguchi, \& Stuart, 2007). Notably, nicotinic synaptic excitation of layer 1 interneurons in auditory cortex may in turn produce inhibition in PV interneurons in layer $2 / 3$, thereby disinhibiting cortical processing during associative learning (Letzkus et al., 2011). The specific nicotinic effects and interactions among interneurons and pyramidal neurons seems to vary among studies, suggesting that effects may vary among cortical regions; such issues will be resolved in future studies.

Other neuromodulatory actions also target distinct interneuron subtypes (Kruglikov \& Rudy, 2008). Fast-spiking (presumed PV) interneurons are modified by activation of muscarinic, serotonin, adenosine and GABA-B receptors. These neuromodulatory actions are presynaptic, and reduce GABA release from the fast-spiking interneurons. Activation of muscarinic receptors produces especially powerful effects, i.e., reduction of feed-forward inhibition. These examples

of neuromodulatory effects clearly demonstrate that inhibitory regulation is itself regulated by other factors. Although most of these studies were performed in neocortical areas other than auditory cortex, neuromodulation undoubtedly alters inhibitory activity in auditory cortex as well.

\section{Conclusion and potential relevance of cortical inhibition to abnormal auditory processing}

Among the abnormal auditory responses observed in schizophrenia is a reduction in the MMN, as noted in the Introduction and elsewhere in this Special Issue (Harms, 2015; Näätänen, Todd, \& Schall, 2015; Wacongne, 2015). The purpose of this review is to promote an understanding of the relationship between the MMN and schizophrenia by highlighting some of the basic cortical circuitry, synaptic interactions and neuronal subtypes that contribute to 
auditory processing. Additional links between basic neurophysiology and clinical use of the MMN are found in recent reviews of stimulus-specific adaptation (SSA), which likely contributes to the production of the MMN (Ayala, et al., 2015; Grimm, et al., 2015). An important conclusion of this review is that while a role for NMDA receptors in generating the MMN has been posited for some time, to concentrate solely on NMDA receptors may be too narrow of an approach. In particular, synaptic regulation of NMDA receptor-mediated activity is a prominent feature of cortical processing, and any mechanistic understanding of the MMN and its dependence on NMDA receptors will need to consider inhibitory regulation as a critical control point.

Notably, nicotine recently has been shown to increase the MMN amplitude in nonsmokers, preferentially in subjects with initially low-amplitude MMNs (Knott et al., 2014). Given nicotine's selective excitation of inhibitory interneurons and the prevalence of smoking among schizophrenics (previous section), it will be interesting to investigate the role of nAChRs in MMN regulation. Synaptic interactions that regulate long-latency activity would likely be involved (e.g., Figs 1-3), since the MMN typically occurs 100-250ms after stimulus onset (Sams, Paavilainen, Alho, \& Naatanen, 1985).

Similar notions apply to abnormal cortical oscillations in schizophrenia, especially oscillations in the gamma range (Uhlhaas, 2011; Williams \& Boksa, 2010). As seen in auditory cortex and other cortical regions, inhibitory interneurons contribute critically to neural oscillations by synchronizing activity among pyramidal neurons, and PV interneurons in particular may contribute to this synchronization (Gonzalez-Burgos \& Lewis, 2012). Inhibitory interneurons also are implicated in pyramidal network oscillations at high gamma frequencies (Suffczynski, Crone, \& Franaszczuk, 2014). Also, as mentioned above, synaptic inhibition appears to be central to stimulus-evoked fluctuations in the gamma band.

Synaptic inhibition tightly regulates the excitatory output of individual neurons and neuronal networks in auditory cortex, as elsewhere. An appreciation of inhibitory regulation will lead to a better understanding of the complexity of auditory processing, including the MMN, which in turn may inform therapeutic approaches to schizophrenia and other disorders. 


\section{References}

Adesnik, H., \& Scanziani, M. (2010). Lateral competition for cortical space by layer-specific horizontal circuits. Nature, 464(7292), 1155-1160.

Ayala, Y. A., Perez-Gonzalez, D., \& Malmierca, M. (2015). Stimulus-specific adaptation in the inferior colliculus: The role of excitatory, inhibitory and modulatory inputs. Biological Psychololgy, this volume.

Broide, R. S., \& Leslie, F. M. (1999). The alpha7 nicotinic acetylcholine receptor in neuronal plasticity. Mol Neurobiol, 20(1), 1-16.

Brosch, M., \& Schreiner, C. E. (1997). Time course of forward masking tuning curves in cat primary auditory cortex. J Neurophysiol, 77(2), 923-943.

Calford, M. B., \& Semple, M. N. (1995). Monaural inhibition in cat auditory cortex. J Neurophysiol, 73(5), 1876-1891.

Catts, V. S., Lai, Y. L., Weickert, C. S., Weickert, T. W., \& Catts, S. V. (2015). Meta-analysis confirms decreased cortical N-methyl-D-aspartate receptor expression levels in schizophrenia: implications for mismatch negativity research. Biological Psychololgy, this issue.

Connors, B. W., Malenka, R. C., \& Silva, L. R. (1988). Two inhibitory postsynaptic potentials, and $\mathrm{GABA}_{\mathrm{A}}$ and $\mathrm{GABA}_{\mathrm{B}}$ receptor-mediated responses in neocortex of rat and cat. J. Physiol. (Lond), 406, 443-468.

Cox, C. L., Metherate, R., Weinberger, N. M., \& Ashe, J. H. (1992). Synaptic potentials and amino acid antagonists in auditory cortex. Brain Res. Bull., 28, 401-410.

Cruikshank, S. J., Urabe, H., Nurmikko, A. V., \& Connors, B. W. (2010). Pathway-specific feedforward circuits between thalamus and neocortex revealed by selective optical stimulation of axons. Neuron, 65(2), 230-245.

DeFelipe, J., Lopez-Cruz, P. L., Benavides-Piccione, R., Bielza, C., Larranaga, P., Anderson, S., ... Ascoli, G. A. (2013). New insights into the classification and nomenclature of cortical GABAergic interneurons. Nat Rev Neurosci, 14(3), 202-216.

Di Cristo, G., Wu, C., Chattopadhyaya, B., Ango, F., Knott, G., Welker, E., . . Huang, Z. J. (2004). Subcellular domain-restricted GABAergic innervation in primary visual cortex in the absence of sensory and thalamic inputs. Nat Neurosci, 7(11), 1184-1186. 
Douglas, R. J., \& Martin, K. A. (1991). A functional microcircuit for cat visual cortex. J Physiol, 440, 735-769.

Ehlers, C. L., Kaneko, W. M., Wall, T. L., \& Chaplin, R. I. (1992). Effects of dizolcilpine (MK801) and ethanol on the EEG and event-related potentials (ERPs) in rats.

Neuropharmacol., 31, 369-378.

Frazier, C. J., Rollins, Y. D., Breese, C. R., Leonard, S., Freedman, R., \& Dunwiddie, T. V. (1998). Acetylcholine activates an alpha-bungarotoxin-sensitive nicotinic current in rat hippocampal interneurons, but not pyramidal cells. J Neurosci, 18(4), 1187-1195.

Gonzalez-Burgos, G., \& Lewis, D. A. (2012). NMDA receptor hypofunction, parvalbuminpositive neurons, and cortical gamma oscillations in schizophrenia. Schizophr Bull, 38(5), 950-957.

Grimm, S., Escera, C., \& Nelken, I. (2015). Early indices of deviance detection in humans and animal models. Biological Psychololgy, this issue.

Gulledge, A. T., Park, S. B., Kawaguchi, Y., \& Stuart, G. J. (2007). Heterogeneity of phasic cholinergic signaling in neocortical neurons. J Neurophysiol, 97(3), 2215-2229.

Hamilton, L. S., Sohl-Dickstein, J., Huth, A. G., Carels, V. M., Deisseroth, K., \& Bao, S. (2013). Optogenetic activation of an inhibitory network enhances feedforward functional connectivity in auditory cortex. Neuron, 80(4), 1066-1076.

Happel, M. F., Jeschke, M., \& Ohl, F. W. (2010). Spectral integration in primary auditory cortex attributable to temporally precise convergence of thalamocortical and intracortical input. J Neurosci, 30(33), 11114-11127.

Harms, L. (2015). Mismatch responses and deviance detection in N-methyl-D-aspartate (NMDA) receptor hypofunction and developmental models of schizophrenia. Biological Psychololgy, this issue.

Javitt, D. C. (2000). Intracortical mechanisms of mismatch negativity dysfunction in schizophrenia. Audiol Neurootol, 5(3-4), 207-215.

Javitt, D. C., Steinschneider, M., Schroeder, C. E., \& Arezzo, J. C. (1996). Role of cortical Nmethyl-D-aspartate receptors in auditory sensory memory and mismatch negativity generation: implications for schizophrenia. Proc Natl Acad Sci U S A, 93(21), $11962-$ 11967. 
Kaczmarek, L. K., \& Levitan, I. B. (Eds.). (1987). Neuromodulation. New York: Oxford University Press.

Kaur, S., Lazar, R., \& Metherate, R. (2004). Intracortical pathways determine breadth of subthreshold frequency receptive fields in primary auditory cortex. J Neurophysiol, 91(6), 2551-2567.

Kepecs, A., \& Fishell, G. (2014). Interneuron cell types are fit to function. Nature, 505(7483), 318-326.

Knott, V., Impey, D., Philippe, T., Smith, D., Choueiry, J., de la Salle, S., \& Dort, H. (2014). Modulation of auditory deviance detection by acute nicotine is baseline and deviant dependent in healthy nonsmokers: a mismatch negativity study. Hum Psychopharmacol, 29(5), 446-458.

Kruglikov, I., \& Rudy, B. (2008). Perisomatic GABA release and thalamocortical integration onto neocortical excitatory cells are regulated by neuromodulators. Neuron, 58(6), 911924.

Letzkus, J. J., Wolff, S. B., Meyer, E. M., Tovote, P., Courtin, J., Herry, C., \& Luthi, A. (2011). A disinhibitory microcircuit for associative fear learning in the auditory cortex. Nature, 480(7377), 331-335.

Levy, R. B., \& Reyes, A. D. (2012). Spatial profile of excitatory and inhibitory synaptic connectivity in mouse primary auditory cortex. J Neurosci, 32(16), 5609-5619.

Li, L. Y., Xiong, X. R., Ibrahim, L. A., Yuan, W., Tao, H. W., \& Zhang, L. I. (2014). Differential Receptive Field Properties of Parvalbumin and Somatostatin Inhibitory Neurons in Mouse Auditory Cortex. Cereb Cortex.

Markram, H., Toledo-Rodriguez, M., Wang, Y., Gupta, A., Silberberg, G., \& Wu, C. (2004). Interneurons of the neocortical inhibitory system. Nat Rev Neurosci, 5(10), 793-807.

McGehee, D. S., \& Role, L. W. (1995). Physiological diversity of nicotinic acetylcholine receptors expressed by vertebrate neurons. Annu Rev Physiol, 57, 521-546.

Metherate, R., \& Ashe, J. H. (1993). Ionic flux contributions to neocortical slow waves and nucleus basalis-mediated activation: whole-cell recordings in vivo. J. Neurosci., 13, 5312-5323. 
Metherate, R., \& Ashe, J. H. (1994). Facilitation of an NMDA receptor-mediated EPSP by paired-pulse stimulation in rat neocortex via depression of GABAergic IPSPs. J. Physiol. (Lond.), 481, 331-348.

Metherate, R., \& Ashe, J. H. (1995). GABAergic suppression prevents the appearance and subsequent fatigue of an NMDA receptor-mediated potential in neocortex. Brain Res., 699, 221-230.

Metherate, R., \& Cruikshank, S. J. (1999). Thalamocortical inputs trigger a propagating envelope of gamma-band activity in auditory cortex in vitro. Exp Brain Res, 126(2), 160-174.

Metherate, R., Kaur, S., Kawai, H., Lazar, R., Liang, K., \& Rose, H. J. (2005). Spectral integration in auditory cortex: Mechanisms and modulation. Hear Res, 206(1-2), 146158.

Näätänen, R., Todd, J., \& Schall, U. (2015). Mismatch negativity (MMN) as biomarker predicting psychosis in clinically at-risk individuals. Biological Psychololgy, this issue.

Pantev, C., Okamoto, H., Ross, B., Stoll, W., Ciurlia-Guy, E., Kakigi, R., \& Kubo, T. (2004). Lateral inhibition and habituation of the human auditory cortex. Eur J Neurosci, 19(8), 2337-2344.

Penttonen, M., Kamondi, A., Acsady, L., \& Buzsaki, G. (1998). Gamma frequency oscillation in the hippocampus of the rat: intracellular analysis in vivo. Eur J Neurosci, 10(2), 718-728.

Pi, H. J., Hangya, B., Kvitsiani, D., Sanders, J. I., Huang, Z. J., \& Kepecs, A. (2013). Cortical interneurons that specialize in disinhibitory control. Nature, 503(7477), 521-524.

Poorthuis, R. B., Bloem, B., Schak, B., Wester, J., de Kock, C. P., \& Mansvelder, H. D. (2012). Layer-Specific Modulation of the Prefrontal Cortex by Nicotinic Acetylcholine Receptors. Cereb Cortex.

Porter, J. T., Cauli, B., Tsuzuki, K., Lambolez, B., Rossier, J., \& Audinat, E. (1999). Selective excitation of subtypes of neocortical interneurons by nicotinic receptors. J Neurosci, 19(13), 5228-5235.

Pouille, F., \& Scanziani, M. (2001). Enforcement of temporal fidelity in pyramidal cells by somatic feed-forward inhibition. Science, 293(5532), 1159-1163.

Purpura, D. P., \& Shofer, R. J. (1964). Cortical intracellular potentials during augmenting and recruiting responses. I. Effects of injected hyperpolarizing currents on evoked membrane potential changes. J. Neurophysiol., 27, 117-132. 
Purpura, D. P., Shofer, R. J., \& Musgrave, F. S. (1964). Cortical intracellular potentials during augmenting and recruiting responses. II. Patterns of synaptic activities in pyramidal and nonpyramidal tract neurons. J. Neurophysiol., 27, 133-151.

Ribaupierre, F. d., Goldstein, M. H., \& Yeni-Komshian, G. (1972). Intracellular study of the cat's primary auditory cortex. Brain Res., 48, 185-204.

Rudy, B., Fishell, G., Lee, S., \& Hjerling-Leffler, J. (2010). Three groups of interneurons account for nearly $100 \%$ of neocortical GABAergic neurons. Dev Neurobiol.

Rugg, M. D., \& Coles, M. G. H. (Eds.). (1995). Electrophysiology of Mind: Event-related brain potentials and cognition (Vol. 25). Oxford: Oxford University Press.

Sams, M., Paavilainen, P., Alho, K., \& Naatanen, R. (1985). Auditory frequency discrimination and event-related potentials. Electroencephalogr Clin Neurophysiol, 62(6), 437-448.

Suffczynski, P., Crone, N. E., \& Franaszczuk, P. J. (2014). Afferent inputs to cortical fastspiking interneurons organize pyramidal cell network oscillations at high-gamma frequencies (60-200 Hz). J Neurophysiol, 112(11), 3001-3011.

Suga, N. (1995). Sharpening of frequency tuning by inhibition in the central auditory system: tribute to Yasuji Katsuki. Neurosci Res, 21(4), 287-299.

Sutor, B., \& Hablitz, J. J. (1989). EPSPs in rat neocortical neurons in vitro. I. Electrophysiological evidence for two distinct EPSPs. J. Neurophysiol., 61, 607-620.

Sutter, M. L., \& Loftus, W. C. (2003). Excitatory and inhibitory intensity tuning in auditory cortex: evidence for multiple inhibitory mechanisms. J Neurophysiol, 90(4), 2629-2647.

Tan, A. Y., Zhang, L. I., Merzenich, M. M., \& Schreiner, C. E. (2004). Tone-evoked excitatory and inhibitory synaptic conductances of primary auditory cortex neurons. J Neurophysiol, 92(1), 630-643.

Todd, J., Whitson, L., Smith, E., Michie, P. T., Schall, U., \& Ward, P. B. (2014). What's intact and what's not within the mismatch negativity system in schizophrenia. Psychophysiology, 51(4), 337-347.

Uhlhaas, P. J. (2011). High-frequency oscillations in schizophrenia. Clin EEG Neurosci, 42(2), $77-82$.

Vianney-Rodrigues, P., Iancu, O. D., \& Welsh, J. P. (2011). Gamma oscillations in the auditory cortex of awake rats. Eur J Neurosci, 33(1), 119-129. 
Wacongne, C. (2015). A predictive coding account of MMN reduction in schizophrenia. Biological Psychololgy, this issue.

Wang, X. J., \& Buzsaki, G. (1996). Gamma oscillation by synaptic inhibition in a hippocampal interneuronal network model. J Neurosci, 16(20), 6402-6413.

Wehr, M., \& Zador, A. M. (2003). Balanced inhibition underlies tuning and sharpens spike timing in auditory cortex. Nature, 426(6965), 442-446.

Wehr, M., \& Zador, A. M. (2005). Synaptic mechanisms of forward suppression in rat auditory cortex. Neuron, 47(3), 437-445.

Williams, S., \& Boksa, P. (2010). Gamma oscillations and schizophrenia. J Psychiatry Neurosci, 35(2), 75-77.

Wu, G. K., Arbuckle, R., Liu, B. H., Tao, H. W., \& Zhang, L. I. (2008). Lateral sharpening of cortical frequency tuning by approximately balanced inhibition. Neuron, 58(1), 132-143.

Xu, X., Roby, K. D., \& Callaway, E. M. (2010). Immunochemical characterization of inhibitory mouse cortical neurons: three chemically distinct classes of inhibitory cells. J Comp Neurol, 518(3), 389-404.

Yuan, K., Fink, K. L., Winer, J. A., \& Schreiner, C. E. (2011). Local connection patterns of parvalbumin-positive inhibitory interneurons in rat primary auditory cortex. Hear Res, 274(1-2), 121-128.

Zhao, Y., Zhang, Z., Liu, X., Xiong, C., Xiao, Z., \& Yan, J. (2015). Imbalance of excitation and inhibition at threshold level in the auditory cortex. Front Neural Circuits, 9, 11. 